\title{
A method for detecting the early stages of local corrosion pro- cesses
}

\author{
Vladimir Blokhin ${ }^{1}$, Anton Dorosinskiy ${ }^{1, *}$, Aleksandr Manjosov ${ }^{1}$, Andrey Markin ${ }^{2}$ \\ ${ }^{1}$ Scientific and production LLC "Sonar", Penza, Russia \\ ${ }^{2}$ Branch of Tyumen industrial University, Nizhnevartovsk, Russia
}

\begin{abstract}
The article discusses the issues of monitoring the parameters of carbon dioxide corrosion of oil and gas production equipment and pipelines. The features of the main control methods traditionally used in the construction of corrosion monitoring systems, both in gas and in oil environments, are described. The article shows the random nature of local corrosion. A new approach to the creation of systems capable of detecting manifestations of local corrosion in real time and corresponding to the concept of "smart" deposits is proposed.
\end{abstract}

\section{Introduction}

Corrosion of the internal surfaces of process equipment at production sites in the oil and gas industry annually leads to significant economic and environmental damage. In the gas industry, carbon dioxide corrosion of steel is a great danger, which has become particularly relevant recently in the development of new oil and gas basins [1]. It is known that a distinctive feature of carbon dioxide corrosion of steel is the possibility of its localization in the form of ulcers, pits or extended grooves along the pipeline bottom (the so-called "streaming" (grooving) corrosion) $[2,3]$.

There is no doubt that corrosion is a complex multifactorial process. To understand it a proper assessment of whole range of different parameters should be taken into consideration. An analysis of the mechanisms of carbon dioxide corrosion of steel shows that its manifestations can be divided into uniform corrosion and localized corrosion (pitting, ulcers, mesa-corrosion, etc.). At the same time, the development of localized corrosion can proceed tenfold faster as compared with the average surface weight loss of the metal [2].

\section{Corrosion prevention methods}

Features of various methods of corrosion monitoring used in oil and gas industry are shown in Table. 1
Table 1. Features of the main methods of corrosion monitoring

\begin{tabular}{|c|c|c|c|c|c|}
\hline № & Method & $\begin{array}{l}\text { Measured } \\
\text { parame- } \\
\text { ter }\end{array}$ & $\begin{array}{l}\text { Moni- } \\
\text { tored } \\
\text { pro- } \\
\text { cesses }\end{array}$ & $\begin{array}{l}\text { Ap- } \\
\text { plica- } \\
\text { bility }\end{array}$ & $\begin{array}{c}\text { Re- } \\
\text { strictions }\end{array}$ \\
\hline 1 & $\begin{array}{l}\text { Weight } \\
\text { loss } \\
\text { coupons } \\
\text { (gravime } \\
\text { tric) }\end{array}$ & $\begin{array}{l}\text { The } \\
\text { change in } \\
\text { mass of } \\
\text { corrosion } \\
\text { coupons } \\
\text { by the } \\
\text { gravimet- } \\
\text { ric method } \\
\text { during the } \\
\text { exposure }\end{array}$ & $\begin{array}{l}\text { Uniform } \\
\text { and } \\
\text { local- } \\
\text { ized } \\
\text { corro- } \\
\text { sion, } \\
\text { erosion. }\end{array}$ & $\begin{array}{l}\text { Any } \\
\text { enviro } \\
\text { nment } \\
\text { s }\end{array}$ & $\begin{array}{l}\text { 1. Internal } \\
\text { ity } \\
2 . \text { Integral } \\
\text { infor- } \\
\text { mation } \\
\text { 3. Impossi } \\
\text { bility of } \\
\text { automa- } \\
\text { tion }\end{array}$ \\
\hline 2 & $\begin{array}{l}\text { Electrica } \\
1 \\
\text { Resistan } \\
\text { ce (ER) }\end{array}$ & $\begin{array}{l}\text { Probe } \\
\text { electrical } \\
\text { resistance }\end{array}$ & $\begin{array}{l}\text { Uniform } \\
\text { corro- } \\
\text { sion, } \\
\text { erosion }\end{array}$ & $\begin{array}{l}\text { Any } \\
\text { enviro } \\
\text { nment } \\
\text { s }\end{array}$ & $\begin{array}{l}\text { Not in- } \\
\text { tended to } \\
\text { measure } \\
\text { local cor- } \\
\text { rosion } \\
\text { rate. }\end{array}$ \\
\hline 3 & $\begin{array}{l}\text { Linear } \\
\text { Polarizat } \\
\text { ion Re- } \\
\text { sistance } \\
\text { (LPR) }\end{array}$ & $\begin{array}{l}\text { Polariza- } \\
\text { tion re- } \\
\text { sistance of } \\
\text { the system } \\
\text { metal- } \\
\text { medium }\end{array}$ & $\begin{array}{l}\text { Uniform } \\
\text { corro- } \\
\text { sion, } \\
\text { pitting } \\
\text { index }\end{array}$ & $\begin{array}{l}\text { Condu } \\
\text { ctive } \\
\text { enviro } \\
\text { nment }\end{array}$ & $\begin{array}{l}\text { The water } \\
\text { content in } \\
\text { the envi- } \\
\text { ronment is } \\
\text { more than } \\
40-50 \%\end{array}$ \\
\hline
\end{tabular}

In a gravimetric method the corrosion rate is calculated by the weight loss of corrosion coupons in the assumption that corrosion is uniform. Measuring the nonuniformity of the mass loss of a metal over the surface of a corrosion coupon (for example, a profilometer), one can not only fix the fact of local corrosion, but also measure its rate. However, the low sensitivity of the gravimetric method, the need for long-term observations, as well as the complexity of methods for assessing local 
corrosion using profilometers virtually eliminates the possibility of its use for the analysis of fast processes.

Due to methodological limitations, ER-sensors also register only average surface corrosion-erosion processes, a method for measuring the thickness of a sensitive element (through a change in electrical resistance) [3].

\section{Analysis of convergence results}

Despite the fact that these methods estimate the same parameter - the rate of average surface metal weight loss - the absolute values of the corrosion rate obtained by each of the above methods often differ from each other. An analysis of the causes of such discrepancies is described in [3], and the conditions for the convergence of the readings of gravimetric and ER sensors are also indicated there (Fig. 1.).

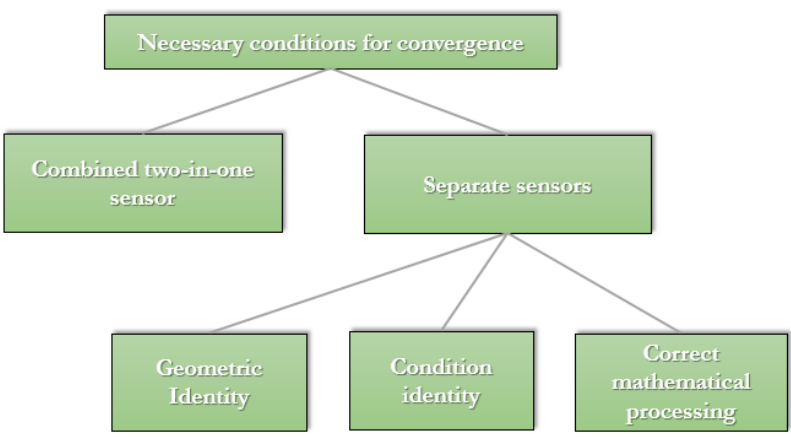

Fig. 1. Conditions of convergence of the results of the weight and ER methods

The greatest convergence is provided in the case when the sensitive element of the ER probe is at the same time a corrosion coupon of two-in-one corrosion (such sensors manufactured by Scientific and production LLC "Sonar" for laboratory testing). Samples of the sensors after testing are shown in Fig. 2

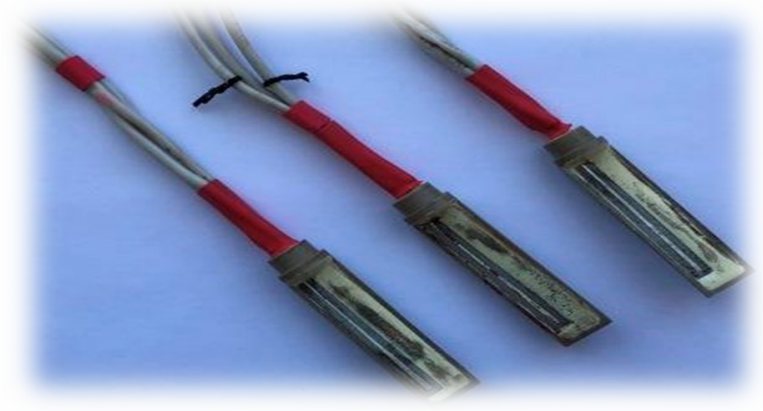

Fig. 2. Photos of universal sensitive elements

Three samples were subjected to corrosion after which the deviation between the results obtained using gravimetric and ER method was recorded. The tests consisted of three phases. At the first stage, the samples were exposed to corrosion for three cycles. In the second and third stages, the effect of corrosion was simulated for two cycles. The duration of each cycle was 96 hours.

The research results confirmed the good convergence of this approach (Fig. 3.).

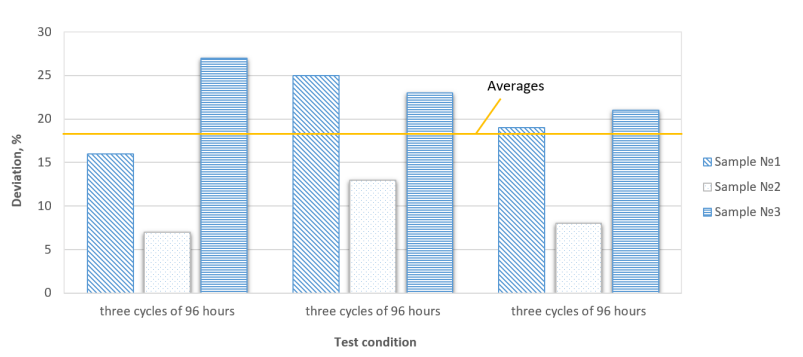

Fig. 3. Test results for universal sensitive elements

Good convergence was observed even in those cases when local corrosion was clearly recorded on the surface of the sensitive elements of the probes.

The development of local corrosion, even with the same test effects, is due to the fact that the metal surface has electrochemical heterogeneity, manifested in the uneven distribution of the potential over the metal surface and the formation of microgalvanopair, which can migrate over the metal surface.

At the macro level, corrosion is expressed in the form of a relatively uniform average surface weight loss of the metal. When one or several microgalvanopairs are fixed on a certain portion of the metal surface, local corrosion develops on this portion. On the fig. 4 photographs of the sensitive elements of the ER-probes (magnification 100 $x$ ) which were subjected to the same corrosive conditions are shown.

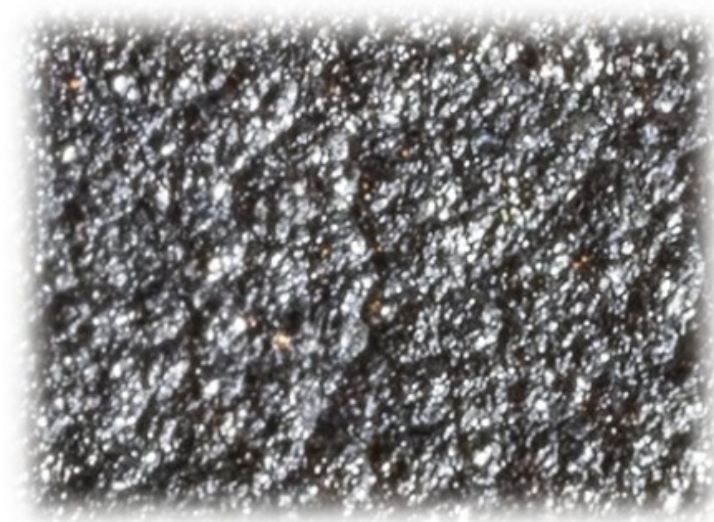

a)

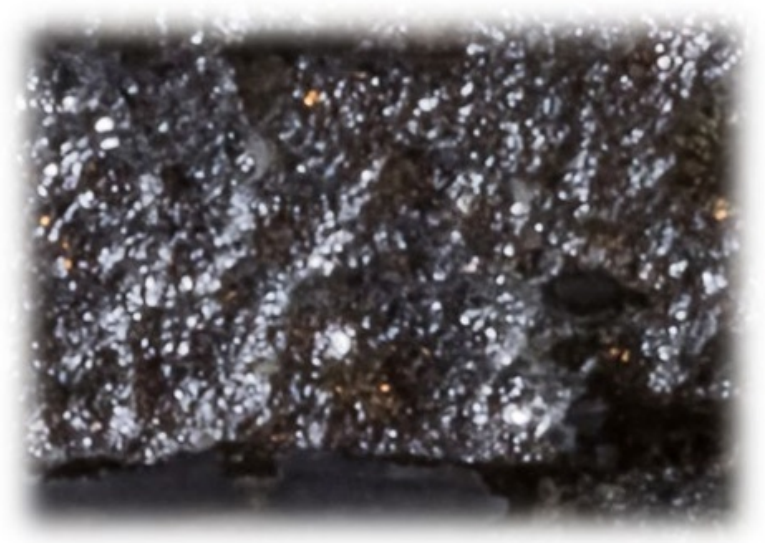

b)

Fig. 4. Photos of the surface of various sensitive elements 
Fig. 4a represents the case when local corrosion is slightly manifested, fig. $4 \mathrm{~b}$ shows the case of a more significant local corrosion manifestation.

Since the electrochemical heterogeneity of the metal surface affects the fixation of micro galvanopairs and is random in nature, it is not possible to predict exactly which part of the metal this will occur.

Based on the foregoing, it is obvious that the process of development of local corrosion not recorded by traditional technical means can lead to the rapid development of its focus and damage to equipment. The prevention of such a situation can be solved with the help of a tool that, in addition to measuring the parameters of the average surface metal loss, detects the nucleation of the local corrosion process and evaluates its characteristics in real time.

\section{Detection of early stages of local cor- rosion}

Taking into account the multi-factor nature of this process, it is advisable to increase the number of monitored parameters when building monitoring systems that describe the current state of the environment from the perspective of the early stages of detection of local corrosion processes.

This approach will allow the observer to see additional parameters of the multidimensional process of carbon dioxide corrosion, which are not visible with the help of the currently used "one-dimensional" tools.

It is possible to expand the number of monitored parameters of the environment due to both the spatial separation of corrosion sensors and the organization of synchronous measurements with instruments operating on different physical principles.

The proposed system (called by us "SyDerCo" - a "streaming" corrosion detection system) is a combined instrument built on the basis of two measuring ER- and LPR channels (for measuring the parameters of polarization resistance). The result of such a system is the determination of the initial phase of carbon dioxide corrosion with the release of information about its parameters.

The idea of this approach is as follows. At one point in the pipeline in close proximity to each other installs:

- Corrosion coupon in the lower part of the pipeline "flush";

- LPR-sensor, designed for operation in environments with low conductivity, which is installed in the lower generatrix of the pipeline "flush";

- ER-sensor, which is also installed in the lower part of the pipeline "flush";

- ER sensor located in the central part of the pipeline (to control the rate of corrosion and erosion wear).

In this case, the corrosion coupon is required for the initial "calibration" of the system by the magnitude of the average surface weight loss of the metal and by the rate of local corrosion. To measure local corrosion with the help of corrosion coupon, additional measurements with optical or needle micrometers, profilometers, etc. are required. Measurement data are justified at the initial stage of system calibration to verify the ER and LPR readings of sensors, adjustment and improvement.

In the absence of a water phase in the lower generatrix of the pipeline, the readings of both ER sensors are close, and the LPR readings of the device register a zero corrosion rate (due to the absence of a water phase, which is the factor "triggering" the local corrosion mechanism). Non-zero LPR sensor readings will indicate the appearance of a conductive medium in the lower generator of the pipeline.

The appearance of the conductive phase is a consequence of the intense condensation of low-mineralized water, which will contribute to the formation and development of a "stream". As its mineralization increases, the LPR sensor will register an increase in corrosion rate. Exceeding the formulated threshold value in conjunction with other parameters will allow fixing the fact of the onset of "streaming" corrosion.

In parallel, an ER sensor installed in the lower tube generates a significant increase in corrosion rate compared to previous values and with ER readings installed in the center of the pipe. If the conditions in the pipeline change, and the low-mineralized water will no longer form a "stream" on the lower generator of the pipeline, this will also be seen from the LPR sensor readings when comparing the readings of two ER sensors.

The use of various methods for measuring the corrosion rate in combination with the zoning of the tube space allows not only to verify the results obtained from the average surface metal loss but also to control the local corrosion parameters based on algorithms using the mathematical apparatus of mutual correlation processing of results.

In this case, the mutual correlation analysis of time series of corrosion rates obtained by various methods is justified by the fact that it allows to reveal hidden patterns based on the expression

$$
(V * W)_{i} \stackrel{\text { def }}{=} \sum_{j}\left(V_{j}^{*} W_{i+j}\right)
$$

where $\mathrm{V}$ and $\mathrm{W}$ - the two time series of corrosion rates studied;

$\mathrm{i}$ - the shift between sequences relative to each other, and the asterisk superscript means complex conjugation.

Verification of the results of measurements of local corrosion parameters is possible by detailed analysis of the corrosion coupon using the methods of profilometry.

The block diagram of the "SyDerCo" system is shown in Fig.5. 


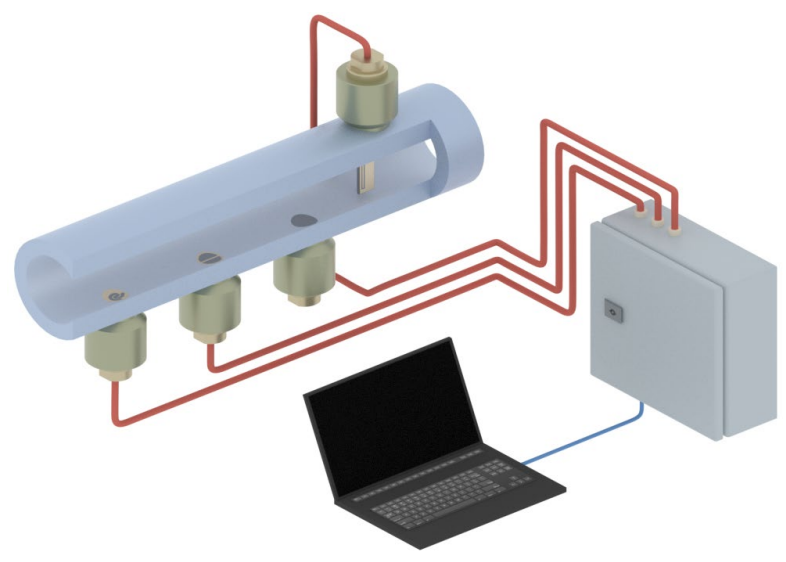

Fig. 5. Block diagram of the system "SyDerCo".

The prototype of the system was built on the basis of commercially available equipment of Scientific and production LLC "Sonar" and was tested on test liquids that simulate in laboratory conditions the actual processes occurring in pipelines.

The presence of such a measuring tool allows you to expand the number of "visible" processes occurring in the in-line space, control them and develop an effective technology of inhibition.

As part of the creation of the "SyDerCo" system, a positive decision was received on issuing a patent for an application for invention No. 2014123875 of $06 / 29 / 18$ "A method for monitoring carbon dioxide corrosion on field pipelines and a device for its implementation."

\section{Conclusions}

1. The application of traditional approaches to the analysis of corrosion in oil and gas environments in the event of a mechanism of carbon dioxide corrosion can lead to the fact that the process of intensive development of local corrosion will not be noticed.

2 . To detect the fact of initiation of the local corrosion process and control its parameters, a new approach to constructing a multiparameter real-time system has been proposed. Practical application of the system "SyDerCo" will allow:

2.1 Increase the efficiency of selection of corrosion inhibitors, taking into account the quality of suppressing losses not only of the average surface mass of the metal, but also of the local component.

2.2 To carry out comprehensive multi-parameter monitoring of carbon dioxide corrosion processes, based on statistically correct, verified data.

2.3 Create a unified corrosion control node, suitable for widespread use in the design of "digital" deposits that exist in real time.

3. The collection of statistics of the system in various real conditions will allow progress in understanding the poorly studied processes of carbon dioxide corrosion and will contribute to the development of new effective inhibitors and the technology of their application.

\section{References}

1. R.V. Kashkovskiy, K.A. Ibatulin, Science and technology in the gas industry, 67 (2016)

2. A.N. Markin, R.E. Nizamov, $\mathrm{CO}_{2}$-Corrosion of oilfield equipment. (JSC "VNIIOENG», 2003)

3. V.A. Blokhin, A.K. Manjosov, A.N. Markin, Corrosion, 39 (2018) 